\title{
Spectrum-aware and Cognitive Sensor Networks for Smart Grid Applications
}

\author{
A. Ozan Bicen, Student Member, IEEE, V. Cagri Gungor, Member, IEEE, Ozgur B. Akan, Senior Member, IEEE
}

\begin{abstract}
Recently, wireless sensor networks (WSN) have been considered as an opportunity to realize reliable and low-cost remote monitoring systems for smart grid. However, interference due to nonlinear electric power equipment and fading as a result of obstacles in various smart grid environments from generation to end-user sides make realization of reliable and energy-efficient communication a challenging task for WSN in smart grid. In this paper, spectrum-aware and cognitive sensor networks (SCSN) are proposed to overcome spatio-temporally varying spectrum characteristics and harsh environmental conditions for WSN-based smart grid applications. Specifically, potential advantages, application areas, and protocol design principles of SCSN are introduced. The existing communication protocols and algorithms devised for dynamic spectrum management networks and WSN are discussed along with the open research issues for the fulfilment of SCSN. A case study is also presented to reveal the reliable transport performance in SCSN for different smart grid environments. Lastly, different energy harvesting techniques for SCSN-based smart grid applications are reviewed. Here, our goal is to envision potentials of SCSN for reliable and low-cost remote monitoring solutions for smart grid.
\end{abstract}

Index Terms-Smart grid, remote monitoring, spectrum-aware and cognitive sensor networks, dynamic spectrum management.

\section{INTRODUCTION}

$\mathbf{S}$ MART grid has been conceived as the evolution of electric power systems to enhance efficiency, reliability and safety of the existing power grid. The need for next-generation electricity network has arisen with the increasing demand for electricity, aging grid equipment, advancement of alternative energy resources, and climate changes to provide reliable, safe and economical power delivery [1]-[4]. To this end, remote and timely information gathering from smart grid equipment about failures, capacity limitations, and natural accidents is extremely crucial for ensuring proactive and realtime diagnosis of possible blackouts and transient faults in the smart grid. This makes cost-effective remote monitoring and control technologies vital for safe, reliable and efficient power delivery in smart grid [5], [6]. An illustrative architecture of the smart grid is shown in Fig. 1.

Recently, wireless sensor networks (WSN), which are mainly characterized by their collaborative, low-cost, and energy-limited nature, have gained attention for electric power

A. O. Bicen and O. B. Akan are with the Next-generation and Wireless Communications Laboratory (NWCL), Department of Electrical and Electronics Engineering, Koc University, Istanbul, 34450 Turkey (e-mail: abicen@ku.edu.tr; akan@ku.edu.tr). V. C. Gungor is with the Computer Networks and Mobile Communications Laboratory, Department of Computer Engineering, Bahcesehir University, Istanbul, 34353 Turkey (e-mail: cagri.gungor@bahcesehir.edu.tr). network monitoring instead of wired systems. Reliable and efficient management of smart grid can be accomplished with the installation of wireless sensor nodes on the critical power grid equipment [5], [6]. Gathered information from this equipment can help in responding to changing conditions and malfunctions of the electric grid in a proactive manner. Moreover, obtained information from sensors can be used to diagnose arising problems rapidly, and hence, autonomous and reliable operation can be achieved in smart grid. However, the realization of smart grid literally depends on the communication capabilities of sensor network in harsh and complex electricity network environments that bring out great challenges for reliability and energy efficiency in WSN.

To this end, dynamic and opportunistic spectrum access capabilities of cognitive radio can be benefited to address many of the unique requirements and challenges of smart grid for WSN, i.e., heterogeneous spectrum characteristics changing over time and space, reliability and latency requirements, harsh environmental conditions, and energy constraints of low-power sensor nodes. With its adaptability to existing spectrum utilization and characteristics in the deployment field, spectrumaware and cognitive sensor networks can enhance the overall network performance and spectrum utilization [7]. Promising advantages of spectrum-aware and cognitive radio equipped sensor nodes in smart grid can be outlined as follows:

- Minimization of environmental effects: Field tests in [5] show that wireless links in smart grid are exposed to spatio-temporally varying spectrum characteristics due to electromagnetic interference, equipment noise, dynamic topology changes, and fading due to obstructions and hindrances. This leads to both time and location dependent delay and capacity variations of wireless links in smart grid environments. Therefore, to overcome varying link conditions in time and space domains, sensor nodes must be capable of reconfiguring themselves autonomously without hardware modifications. With the ability of dynamic and opportunistic access to spectrum, sensor nodes can mitigate these effects while minimizing energy consumption.

- Access to licensed and unused spectrum bands: Different services operating in licensed bands of spectrum can be accessed by users in smart grid. Cognitive radio capability empowers sensor nodes to detect spectrum holes and access them without interfering with licensed users. Therefore, cognitive radio equipped sensor nodes can dynamically access vacant bands based on spectrum opportunities, and achieve higher capacity levels with the 


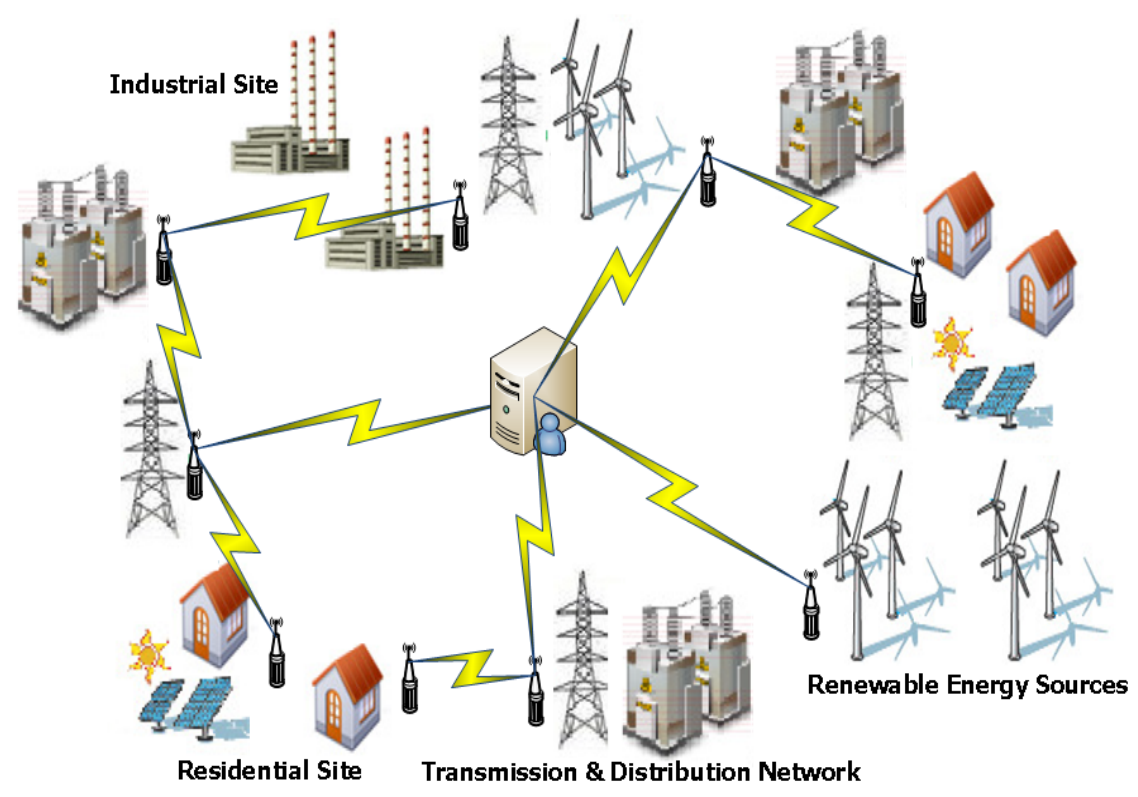

Fig. 1. An illustrative architecture of smart grid using sensor networks. Smart grid can be defined as a modernized electric power T\&D network using robust two-way communications, advanced sensors, and distributed computing technologies to improve efficiency, reliability and safety of power delivery and use.

same amount of power consumption.

- Adaptation to different spectrum utilization patterns: Smart grid is distributed over a large geographic area, and different spectrum utilization patterns can be experienced in these areas. Sensor nodes equipped with cognitive radio can continue reporting of sensed phenomenon under different spectrum characteristics. Therefore, cognitive capability does not only increase overall spectrum utilization, but also alleviate adaptation to different spectrum utilization patterns in smart grid as well.

- Overlay deployment of multiple sensor networks: The existing WSN deployments are based on static spectrum apportionment. Since multiple nodes from different sensor networks try to send its information simultaneously, sensor nodes are subject to interference and collisions. Cognitive radio can provide efficient spectrum sharing between co-existing sensor networks in a fair manner.

In general, spectrum-aware and cognitive sensor networks (SCSN) for smart grid can be specified as a distributed wireless network of spectrum-aware sensor nodes, which monitor the critical smart grid equipment and send their information dynamically over available spectrum bands in a multi-hop fashion to meet the application-specific requirements. Albeit the recent interest in power grid monitoring based on WSN, the SCSN for smart grid is a vastly unexplored area. To the best of our knowledge, there exist no comprehensive work on employment of cognitive radio sensor networks for smart grid applications. In addition to study of potential applications and communication algorithm needs of SCSN, in this paper we also investigate incorporation of energy harvesting techniques for different power grid segments to extend limited life time of sensor nodes. Here, our objective is to envision potentials of SCSN for smart grid applications and discuss the open research issues in this timely and exciting field.

The remainder of the paper is organized as follows. In
Section II, the potential applications of SCSN in smart grid are briefly discussed. The spectrum management functionalities in SCSN are presented in Section III. The specific technical challenges and open research directions for the communication layers of SCSN are summarized, and reliable transport performance in SCSN for various smart grid environments is asses via a case study in Section IV. Different energy harvesting solutions for SCSN-based smart grid applications are reviewed in Section V. Finally, the paper is concluded in Section VI.

\section{Potential Applications of SCSN in Smart GRid}

WSN has already started to be used in a diverse range of power grid applications from home area networks to power transmission and distribution (T\&D) network monitoring [1], [5], [6], [8], [9]. In Table I, an overview of some of these potential applications and their corresponding power grid segment are given [1], [5], [8], [6], [9]. In general,the electric power systems contain three major subsystems, power generation, T\&D, and consumer facilities.

\section{A. Remote Monitoring for Electric Power Generation Systems}

A wide variety of sensors, such as current, voltage, temperature, etc., have been used in conventional electric power generation systems (EPGS). Lately, green energy resources, such as wind power and solar energy, have been gaining popularity in electricity generation, since the green electricity generation techniques meet environmental regulations by producing lower carbon emission in contrast to conventional EPGS. Due to variations and limitations on the availability of solar and wind power, real-time information obtained from green EPGS is crucial for electrical energy storage units.

Recently, employing wireless sensors in EPGS has been an active research field due to the collaborative nature and flexibility of WSN [8]. However, realization of wireless remote 
TABLE I

Summary of SCSN APPLICATIONS FOR SMART GRID vs. POWER GRID SEgMENT

\begin{tabular}{cc} 
Applications & Power Grid Segment \\
\hline \hline Wireless Automatic Meter Reading & Consumer Side \\
Real Time Pricing and Demand Response & Consumer Side \\
Residential Energy and Load Management & Consumer Side \\
\hline Line Fault and Power Theft Detection & T\&D Side \\
Outage Detection & T\&D Side \\
Underground Cable System Monitoring & T\&D Side \\
Towers and Poles Monitoring & T\&D Side \\
Animals and Vegetation Control Monitoring & T\&D Side \\
Conductor Temperature and Dynamic Thermal Rating Systems & T\&D Side \\
\hline Traditional Power Plant Monitoring & Generation Side \\
Wind Farm Monitoring & Generation Side \\
Solar Farm Monitoring & Generation Side
\end{tabular}

monitoring applications in EPGS is challenging due to the crowded spectrum problem in unlicensed bands. Moreover, performance of WSN in smart grid is severely restricted by packet losses, collisions and contention delays due to spatiotemporally varying heterogenous spectrum characteristics [10]. In this regard, the SCSN nodes can access the spectrum opportunistically to improve the overall network performance in terms of reliability and communication latency. In addition to remote monitoring of EPGS equipment, SCSN can also be deployed for monitoring the workspace for the safety of staff.

\section{B. Remote Monitoring for Electricity T\&D Network}

Transmission and distribution (T\&D) power networks are very critical power grid assets, where an equipment failure can result in electricity blackouts and various accidents. In T\&D equipment monitoring, several variables, such as temperature, conductor thermal capacity, faulted circuit indicators, conductor sag, conductor vibration, can be monitored [6]. Since transmission power lines are distributed over a large geographic area, different spectrum utilization patterns are exposed in these areas. Cognitive capability helps to adapt different spectrum patterns in smart grid easily. Substation monitoring and control is also important for the power grid [6]. The SCSN nodes can overcome challenges due to electromagnetic interference and fading in substations with their spectrum-aware nature. Moreover, the SCSN can achieve higher throughput with its DSA capability, and hence, surveillance of power lines and substations can be achieved with enhanced realtime communication capability of the SCSN, and bandwidthgreedy multimedia applications can be realized, e.g., power grid infrastructure security via video surveillance in smart grid.

\section{Remote Monitoring for Consumer Facilities}

Unlike the conventional power grid, effective power demand control of consumer facilities can be achieved in smart grid. With the opportunistic spectrum access capability of SCSN, monitoring of consumer facilities' power consumption can be incorporated into smart grid without interfering with the existing communication infrastructure at the consumer side. Moreover, DSA capability can help to overwhelm environmental interference and fading. Based on gathered information from different users with various power consumption characteristics, such as industrial and home users, predictive and robust electrical power load balancing strategies can be developed. [5].

As part of the end-user facilities, advanced metering infrastructures (AMI) can also be efficiently realized with the use of the SCSN. The SCSN can contribute to AMI technology for self-configuration and easy deployment in co-existing wireless networks at different customer premises. With the spectrumaware communication capability, AMI meters and equipment can be easily deployed at the remote sides to achieve seamless and reliable communication between utility control center and AMI. The SCSN nodes designed with consideration of energy and price limitations in remote monitoring can be the main components for efficient realization of wireless AMI.

\section{SPECTRUm MANAGEMENT REQUiREMENTS AND CHALlenges OF SCSN}

Minimization of environmental effects, adaptation to different spectrum utilization patterns, and overlay deployment of multiple sensor networks are some of the promising advantages of SCSN in smart grid. However, the realization of SCSN for smart grid mainly requires efficient spectrum management functionalities to dynamically manage the spectrum access of sensor nodes in harsh smart grid environments. Requirements and research challenges for main four spectrum management functionalities in cognitive radio, i.e., spectrum sensing, spectrum decision, spectrum sharing, and spectrum mobility, are explored below for SCSN.

\section{A. Spectrum Sensing}

To take advantage of spectrum sensing in the SCSN, an efficient solution is needed considering both sensor network resource limitations and DSA network challenges. Considering high numbers of sensor nodes in large-scale smart grid systems and low-cost requirements, it may not be feasible to equip sensor nodes with multiple radios and highly capable processors. Therefore, sophisticated spectrum sensing algorithms cannot be used. Spectrum sensing should be performed with limited node hardware, possibly using single radio. Assuming that deployed sensor nodes in smart grid environments have single radio due to their scalability and low-cost requirements, 
sensing durations should be minimized as much as possible with the consideration of possible transmission activities and energy efficiency. There are various spectrum sensing methods, such as energy detection, feature detection, matched filter, and interference temperature [11]. Incorporating of one (or hybrid) of these techniques, detection of dynamically changing noise components in smart grid, and modeling of their interference with respect to time and space can be achieved.

Overall, the benefits of DSA, such as lower packet collisions due to the capability of switching to the best available channel, less contention delay and more bandwidth, come with the additional energy consumption caused by spectrum sensing and distribution of these sensing results. The trade off between energy efficiency and sensing accuracy is should be addressed and a detailed analysis of cost vs. benefits for a specific smart grid environment should be performed.

\section{B. Spectrum Decision}

With the DSA capability, sensor networks have ability to change their operating spectrum band when they decide that communication can be done in an another band with increased efficiency and QoS. Selecting one radio frequency as network-wide cannot yield the expected performance gain due to spatio-temporally varying spectrum characteristics. For example, power grid equipment may work periodically, since they may not be required to always work, and hence, their RF interference can also vary with time. In addition, some licensed band users, such as TV and cellular phones, may exist around, especially in consumer sites.

Parameter selection is crucial for efficiency of spectrum decision. These parameters include but not limited to spectrum sensing duration to data transmission ratio, transmission power, expected duration to spend in a channel without spectrum handoff, predictive capacity and delay, energyefficiency and error rate. For underlay approaches and existing smart grid equipment RF interference, the trade-off between spectrum handoff and adaptation to ongoing channel must be investigated. Overall, this yields an optimization problem as to handoff or not to handoff based on channel conditions.

In distributed coordination approaches for spectrum decision, sensor nodes sense the radio spectrum and communicate their spectrum sensing and decision results to their neighbors in order to overcome the spectrum decision problems caused by the limited knowledge of spectrum availability and network topology [11]. Overall, energy efficient and scalable methods of spectrum decision mechanisms are yet to be investigated in order to efficiently realize the proposed SCSN for smart grid.

\section{Spectrum Sharing}

The transmissions in smart grid environments should be coordinated by spectrum sharing functionality to prevent packet collisions and multi-user colliding in crowded radio spectrum environments of the smart grid [11]. Importantly, different applications in smart grid may co-exist, and to satisfy their reliability and latency requirements, QoS-aware spectrum sharing schemes are essential for the proposed SCSN. To achieve this objective and thus to overcome dynamically varying spectrum characteristics, the temporal and spatial reuse of spectrum must be benefited. In general, spectrum sharing functionality is closely related to Medium Access Control (MAC) layer functionality and thus, it can be incorporated into the MAC layer. Some of the main challenges against efficient spectrum sharing schemes include time-synchronization, distributed power allocation and spectrum utilization, and topology discovery. Overall, an effective spectrum sharing scheme helps to meet QoS requirements of smart grid applications by allocating network resources adaptively.

\section{Spectrum Mobility}

In SCSN for smart grid, spectrum handoff (or mobility) can be triggered by excessive interference caused by smart grid equipment. In case of excessive RF interference and noise, ongoing communication should be carried onto an another channel selected by spectrum decision algorithm. To have effective spectrum mobility functionality, the trade-offs between communication parameters must be well understood. Moreover, since spectrum mobility brings interruptions to ongoing communication, the schemes to prevent buffer overflows and minimize communication delay should be developed in order to allow reliable and real-time remote monitoring in SCSN. Based on changes in spectrum characteristics in time and space domains, spectrum handoff can be performed heterogeneously, and hence, this will yield heterogeneous link conditions on the way to sink node. Since smart grid is spread over a large geographic area, spectrum mobility functionality is also critical for adapting to different spectrum regulations.

\section{SCSN COMmunication Protocol Suite}

Efficient operation of the proposed SCSN is tightly-coupled by the running communication protocol suite. In addition to dynamic spectrum access, dense deployment, event driven nature and energy efficiency concerns of the SCSN, harsh environmental conditions and variable link capacity arises in smart grid environments. In this section, the SCSN specific challenges for communication layers are briefly investigated due to space limitations, and then, adaptive reliability control in SCSN is addressed.

\section{A. Physical Layer}

To overcome spatio-temporally varying spectrum characteristics and fading, SCSN node's physical layer must be configurable in terms of operating frequency, modulation, channel coding, transmission power and spectrum sensing duration. This configuration should be based on spectrum sensing and decision results. Due to resource-limited nature and low-cost requirement of sensor nodes, implementing RF front-end for SCSN node is a challenging task. Importantly, SCSN physical layer must be capable of providing statistical information about channel conditions to upper layers for empowering spectrum-awareness. Therefore, effective, energy-efficient, and yet practical cognitive radio for sensor nodes is essential for the realization of SCSN. Existing fundamental open research issues for the realization of physical layer of SCSN can be outlined as follows: 
- In order to overwhelm temporally and spatially varying environmental RF interference in smart grid, adaptive power allocation schemes are essential. Interference problem due to dense deployment in sensor networks should also be considered. Designed solution must maximize energy usage efficiency.

- Adaptive modulation should be employed to maximize network life time and map application-specific QoS requirements to configurable parameters of the physical layer. SCSN node's physical layer must be configurable without hardware modification. Thus, software-defined radios ensuring efficient DSA are needed for SCSN.

- Cooperative transmission schemes must be investigated to benefit from sender diversity in SCSN. Cooperative relaying can help to realize energy-efficient communication in such a harsh RF interference environment as SCSN.

- Statistical methods are required to help channel information gathering in physical layer. Considering limited processing capabilities and low-cost requirements of sensor nodes, practical signal processing algorithms should be developed to enable effective spectrum management, and spectrum awareness at upper layers.

\section{B. Data Link Layer}

Efficient medium access control (MAC), and error control are the main functionalities of the data link layer. In the proposed SCSN, these objectives must be achieved in an energy-efficient manner with consideration of dynamic spectrum management challenges. We investigate MAC and error control in SCSN separately as follows:

1) Medium Access Control: Resource limitations, dense deployment, and application-specific QoS requirements of sensor networks are exacerbated by spatially and temporally varying channel conditions in smart grid. Furthermore, event estimation, spectrum sensing, and channel identification requirements should be considered jointly to determine sleep schedules to reduce energy consumption in the network.

Additional challenges that MAC layer in SCSN must handle to empower DSA are outlined as follows:

- Solutions with minimum control overhead and no additional hardware requirements, such as an additional transceiver, should be developed.

- Joint consideration of spectrum sensing and duty cycling is required to balance the trade-off between energy efficiency and spectrum efficiency.

- Novel spectrum-aware MAC protocol should be developed. It must jointly consider spatial correlation of sensed phenomenon, energy efficiency requirement of sensor nodes, and contention due to dense deployment.

2) Error Control: With its multiple channel access ability, and dynamically varying spectrum conditions, a fixed FEC scheme may not yield optimal results for every channel. Hence, the error correction mechanism must consider this trade-off and adaptive FEC schemes or hybrid ARQ mechanisms can be employed. Enabling spectrum-aware energyefficient error control mechanisms can be made possible with the consideration of the following additional challenges.
- Cooperative schemes based on ARQ can be developed to help lost packets recovery. Nodes receiving the transmitted information can keep this information for a while although they are not the destination node, and can retransmit based on loss prediction or ARQ. Moreover, cooperative relaying schemes may be employed by relay nodes to increase probability of successful packet forwarding. However, efficient synchronization of sensor nodes is required in this case.

- Repetitive ARQs due to harsh smart grid environmental interference can block packet forwarding and can cause congestion due to excessively incoming packets. Added redundancy by FEC employment must be spectrum-aware and predictive such that it must adapt spatially and temporally varying environmental interferences, and must rapidly react to spectrum handoffs.

- Cost vs. benefit analysis of employing FEC, ARQ, hybrid, and cooperative schemes should be well investigated with consideration of spectrum handoff and licensed user activity to provide energy conservation maximization.

\section{Routing Layer}

DSA capability provides interference minimizing opportunity in route selection through the sink node. Moreover, spectrum sensing durations are limiting factor for the throughput of sensor nodes. Moreover, in order to detect variations in the environmental interference, spectrum sensing durations also vary depending on spatially varying characteristics of RF interference sources on the path to sink. Open research issues in SCSN for routing layer are stated below.

- Multi-path routing can be employed to benefit from path diversity for interference mitigation.

- Cooperative routing schemes, such as diffusion-based cooperative routing protocols, can be developed to increase energy efficiency on packet forwarding.

- Depending on spatial variation of environmental interference, different channels can be benefited for forwarding on the event-to-sink path. To this end, spectrum-aware multi-channel routing algorithm can be developed that is maximizing spectrum usage efficiency while minimizing exposed event-to-sink delay.

- Moreover, spectrum decision mechanism should be in contact with routing layer, i.e., spectrum decisions must be performed after investigation of trade-off between spectrum handoff and adaptation of routing layer to concurrent operating channel.

\section{Transport Layer}

Reliability and congestion control become an extremely challenging task with integration of cognitive radio and sensor networks [10], [12]. In SCSN, congestion control algorithms must be aware of cognitive-cycle, and should perform load balancing in a distributed manner. Spatially and temporally varying lossy links makes rate control a very challenging task, and requires careful design for congestion avoidance. Moreover, control packet exchanges must be minimized and congestion control mechanism must be proactive to help 


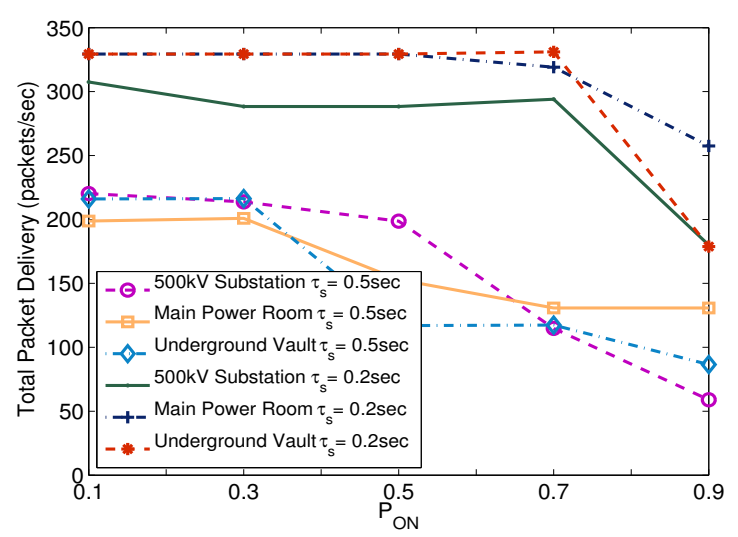

(a)

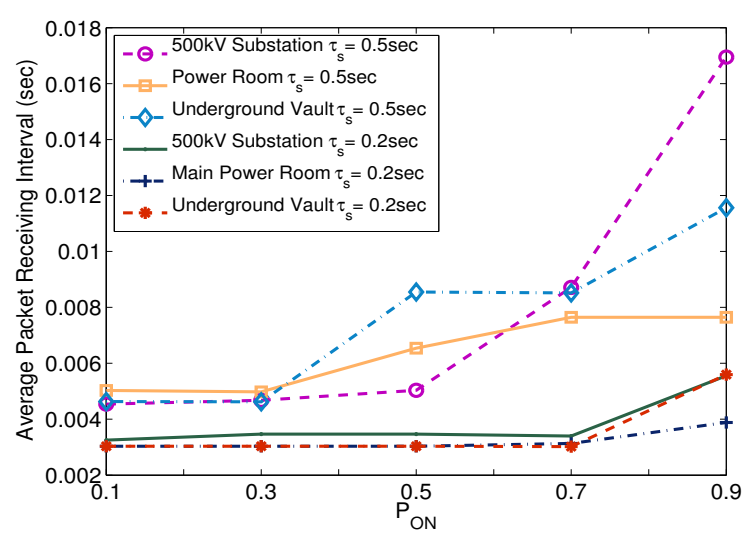

(b)

Fig. 2. Variation of (a) packet delivery and (b) packet receiving interval with respect to licensed user ON probability $\left(P_{O N}\right)$.

TABLE II

LOG-NORMAL SHADOWING PATH LOSS MODEL PARAMETERS FOR DIFFERENT SMART GRID ENVIRONMENTS

\begin{tabular}{c|ccc}
$\begin{array}{c}\text { Power System } \\
\text { Environment }\end{array}$ & $\begin{array}{c}\text { Path Loss } \\
(\eta)\end{array}$ & $\begin{array}{c}\text { Shadowing } \\
\text { Deviation }(\sigma)\end{array}$ & $\begin{array}{c}\text { Noise } \\
\text { Level }(\mathrm{dBm})\end{array}$ \\
\hline 500 kV Substation & 2.42 & 3.12 & -93 \\
\hline Main Power Room & 1.64 & 3.29 & -88 \\
\hline $\begin{array}{c}\text { Underground Network } \\
\text { Transformer Vault }\end{array}$ & 1.45 & 2.45 & -92
\end{tabular}

energy harvesting. Furthermore, in SCSN, environmental RF interference further amplifies the challenges emerging from union of those. Open research directions for reliability and congestion control in SCSN are summarized as follows.

- Statistical rate control schemes must be developed to provide reliable event transport under varying spectrum characteristics and spectrum sensing durations. Obviously, rate control algorithm must not aim to maximize rate and reach maximum bandwidth utilization, instead it must aim to maximize reliability.

- Furthermore, real-time requirements of time-critical applications should be considered, as well as maximizing reliability, real-time transport protocols must minimize delay. This requires additional information at source nodes to determine event to sink path characteristics, and apply rate control to minimize delay while satisfying reliability.

\section{E. A Case Study of Reliable Transport in Different Smart Grid Environments}

We have performed simulation experiments to reveal the reliability performance of cognitive radio equipped sensor nodes in smart grid [10]. Wireless channel model and parameters that are determined in our previous study via field-test experiments in different spectrum environments of power grid, e.g., $500 \mathrm{kV}$ substation, main power room and underground network transformer vaults [5], are used in simulations. Experimentally determined log-normal channel parameters for different power system environments are given in Table II. 200 nodes and a sink are placed randomly in a $100 \mathrm{~m} \times 100 \mathrm{~m}$ field. 10 source nodes are randomly selected within an event area of radius $15 \mathrm{~m}$, and transmit power $\left(P_{t}\right)$ is set to $10 \mathrm{dBm}$. Sensor nodes activity pattern with time consists of data transmission $\left(\tau_{t}\right)$, spectrum sensing $\left(\tau_{s}\right)$ and spectrum handoff $\left(\tau_{h}\right)$ intervals. As licensed user communication is detected, accessed channel is vacated and spectrum handoff is performed. For smart grid environment, licensed user activity can be perceived as wireless channel conditions that restricts communication of sensor nodes. TFRC [13] is used as transport protocol in the simulation experiment, and packet size is limited to 100 bytes. 10 channels are created for each power grid spectrum environment, e.g., $500 \mathrm{kV}$ substation, main power room and underground network transformer vault, given in Table II.

In Fig. 2 (a) and (b), to gain more insight regarding the challenges of reliable transport in SCSN, comparative performance evaluations in terms of packet delivery rate and average packet receiving interval are presented, respectively. Increase in the spectrum sensing duration caused a downswing in the packet delivery performance about $30 \%$ for each environment. Moreover, with the increasing $P_{O N}$ packet delivery performance decays. Average packet receiving interval increases four times with increasing licensed user activity. Extended simulations and their detailed discussions for delay-sensitive and multimedia communications can be found in [10].

\section{ENERGY HARVESTING IN SCSN}

While communicating sensor node's power consumption is on the order of a few milliwatts, it reduces to a few microwatts in sleeping periods. In addition to transmission distance, power consumption of SCSN nodes alter based on the spectrum conditions and different factors, such as employed frequency band, environmental noise, licensed user interference limitations, and spectrum sharing policies with other unlicensed networks. Since battery maintenance and wiring for densely deployed sensor network in smart grid is not a feasible solution, extending battery lifetime is a significant challenge. Meanwhile, mains power might be available in some of the WSN-based smart grid applications, such as in residential energy management. However, sensor nodes, which are deployed in a high voltage smart grid environments, will 
TABLE III

SumMARY OF EXISTING ENERGY HARVESTING TECHNIQUES FOR SCSN

\begin{tabular}{c|ccc}
$\begin{array}{c}\text { Energy Harvesting } \\
\text { Technique }\end{array}$ & Effect & Energy & Principle \\
\hline Magnetic Induction & Electromagnetic & Current passing lines & Magnetic fields \\
Modulated Backscattering & Wave backscattering & RF source & Sender modulates received wave \\
Piezoelectric & Piezoelectric materials & Vibrations & Deformation of piezoelectric materials \\
Pyroelectric & Pyroelectric materials & Heat & Temperature increase or decrease \\
Thermoelectric & Seebeck effect & Heat & Thermal gradients \\
Solar & Photovoltaic effect & Sun & Cells with reverse biased $p n^{+}$junction \\
Electrostatic & Mechanical & Vibrations & Oscillation based varactor plate movements
\end{tabular}

still need appropriate power sources. Recently, a few energy harvesting techniques have been introduced [14]. Energy harvesting can enhance the performance of the SCSN with the self-charging, i.e., self-healing, capability. Unattended energy in the environment, such as, solar, mechanical, thermal, and magnetic, can be scavenged to energize sensor nodes. Possible energy harvesting techniques for SCSN are summarized in Table III, and explained below:

- Magnetic induction: Generated magnetic field by the AC current carrying power lines can be used to induce electric current, and hence, power for sensor nodes. Sensors nearby power lines can benefit from magnetic flux linkage opportunity, and harvested energy can be used for battery recharging purposes.

- Modulated backscattering: Recently, modulated backscattering is proposed for sensor networks, in which radiated wave is backscattered by source node, and modulated accordingly [15]. With modulated backscattering, only receiver node is required to consume power, since source node only modulates the received signal. If receiver node has battery charging opportunity, such as a node that has energy harvesting opportunity and capability by magnetic induction, network lifetime can be greatly extended by the employment of modulated backscattering at source node.

- Other energy harvesting opportunities: Apart from magnetic induction and modulated backscattering, there exists piezoelectric, pyroelectric, thermoelectric, solar, and electrostatic energy harvesting techniques that can also be employed in SCSN for smart grid. While solar harvesting techniques can only applicable to sensors placed on outdoor equipment, pyroelectric and thermoelectric are applicable to sensors placed on both outdoor and indoor equipments. Piezoelectric and electrostatic harvesting techniques exploit mechanical strain deformation and vibration motion to acquire electrical energy, respectively.

\section{CONCLUSION}

Recent field tests show that, reliable communication in smart grid is a challenging task for WSN-based smart grid applications due to electromagnetic interference, equipment noise, dynamic topology changes, and fading. In this paper, spectrum-aware and cognitive sensor networks (SCSN) is introduced to provide reliable and efficient communication for remote monitoring applications in smart grid. First, SCSNbased applications explored for power generation systems,
T\&D networks, and consumer facilities. Then, the challenges and requirements of spectrum management functionalities, i.e., spectrum sensing, spectrum decision, spectrum sharing, and spectrum mobility, are discussed from the perspective of SCSN. The communication protocol suite is discussed from the perspective of SCSN, while putting emphasis on open research directions. A case study is presented to uncover the reliable transport performance in SCSN for various smart grid environments. Lastly, different energy harvesting techniques for SCSN-based smart grid applications are reviewed. We have provided a contemporary perspective to the current state of the art in remote monitoring and control of smart grid via SCSN.

\section{ACKNOWLEDGEMENTS}

The work of A. O. Bicen and V. C. Gungor was supported by the European Union FP7 Marie Curie International Reintegration Grant (IRG) under Grant PIRG05-GA-2009249206. The work of O. B. Akan was supported in part by the Turkish Scientific and Technical Research Council under grant \#110E249 and by the Turkish National Academy of Sciences Distinguished Young Scientist Award Program (TUBA-GEBIP).

\section{REFERENCES}

[1] S. M. Amin and B. F. Wollenberg, "Toward a Smart Grid," IEEE Power and Energy Mag., vol. 3, no. 5, pp. 34-41, Sep./Oct. 2005.

[2] A. Bose, "Smart Transmission Grid Applications and Their Supporting Infrastructure," IEEE Trans. on Smart Grid, vol. 1, no. 1, pp. 11-19, Apr. 2010.

[3] H. Farhangi, "The Path of the Smart Grid," IEEE Power and Energy Mag., vol. 8, no. 1, pp. 18-28, Jan.-Feb. 2010.

[4] C. H. Hauser, D. E. Bakken, and A. Bose, "A Failure to Communicate: Next Generation Communication Requirements, Technologies, and Architecture for the Electric Power Grid," in IEEE Power and Energy Mag., vol. 3, pp. 47-55, Mar.-Apr. 2005.

[5] V. C. Gungor, B. Lu, G.P. Hancke, "Opportunities and Challenges of Wireless Sensor Networks in Smart Grid," IEEE Trans. on Industrial Electronics, vol. 57, no. 10, pp. 3557-3564, Oct. 2010.

[6] Y. Yang, F. Lambert, and D. Divan, "A Survey on Technologies for Implementing Sensor Networks for Power Delivery Systems," in Proc. IEEE Power Engineering Society General Meeting, 2007.

[7] O. B. Akan, O. B. Karli, and O. Ergul, "Cognitive Radio Sensor Networks," IEEE Network, vol. 23, no. 4, pp. 34-40, July-August 2009.

[8] M. Erol-Kantarci, H. T. Mouftah, "Wireless Multimedia Sensor and Actor Networks for the Next-Generation Power Grid," to appear in Elsevier Ad Hoc Networks, 2011.

[9] R. A. Leon, V. Vittal, G. Manimaran, "Application of Sensor Network for Secure Electric Energy Infrastructure," in IEEE Trans. on Power Delivery, vol. 22, no, 2, pp. 1021-1028, 2007.

[10] A. O. Bicen, V. C. Gungor, and O. B. Akan, "Delay-sensitive and Multimedia Communication in Cognitive Radio Sensor Networks," Elsevier Ad Hoc Networks, to appear in 2011, doi:10.1016/j.adhoc.2011.01.021. 
[11] I.F. Akyildiz, W-Y. Lee, and K. R. Chowdhury, "Spectrum Management in Cognitive Radio Ad Hoc Networks," IEEE Network, vol. 23, no. 4, pp. 6-12, July 2009.

[12] A. O. Bicen, and O. B. Akan, "Reliability and Congestion Control in Cognitive Radio Sensor Networks," Elsevier Ad Hoc Networks, vol. 9, no. 7, pp. 1154-1164, Sept. 2011.

[13] S. Floyd, M. Handley, J. Padhye, and J. Widmer, "Equation-based Congestion control for unicast applications," in Proc. ACM SIGCOMM 2000, pp. 45-58, Aug. 2000.

[14] R. Moghe, Yi Yang; F. Lambert, and D. Divan, "A scoping study of electric and magnetic field energy harvesting for wireless sensor networks in power system applications," in Proc. IEEE ECCE 2009, pp. 3550-3557, Sept. 2009.

[15] O. B. Akan, M. T. Isik, and B. Baykal, "Wireless Passive Sensor Networks," IEEE Commun. Mag., vol. 47, no. 8, pp. 92-99, Aug. 2009.

A. Ozan Bicen (abicen@ku.edu.tr) received his B.Sc. degree in electrical and electronics engineering from Middle East Technical University, Ankara, Turkey, in 2010. He is currently a research assistant at Next-generation and Wireless Communications Laboratory (NWCL) and pursuing his M.Sc. degree at the Electrical and Computer Engineering Department, Koc University, Istanbul, Turkey. His current research interests include design and analysis of cognitive radio networks.

V. Cagri Gungor (cagri.gungor@bahcesehir.edu.tr) received his Ph.D. degree in electrical and computer engineering from the Broadband and Wireless Networking Laboratory, Georgia Institute of Technology, Atlanta, GA, USA. Currently, he is the Co-Director of the Computer Networks and Mobile Communications Lab and the Graduate Programs (Ph.D. and M.S.) Coordinator at the Department of Computer Engineering, Bahcesehir University, Istanbul, Turkey. Before joining to Bahcesehir University, he was working at Eaton Corporation, Innovation Center, WI, USA as a Project Leader. His current research interests are in smart grid communications, next-generation wireless networks, wireless ad hoc and sensor networks, cognitive radio networks, and IP networks. Dr. Gungor has authored several papers in refereed journals and international conference proceedings, and has been serving as a reviewer and program committee member to numerous journals and conferences in these areas. He is also the recipient of the IEEE ISCN 2006 Best Paper Award, the European Union FP7 Marie Curie IRG Award in 2009, Turk Telekom Research Grant Awards in 2010 and 2012, and the San-Tez Project Awards supported by Alcatel-Lucent Teletas and the Turkish Ministry of Science, Industry and Technology in 2010.

Ozgur B. Akan (akan@ku.edu.tr) received B.S. and M.S. degrees in electrical and electronics engineering from Bilkent University and METU in 1999 and 2001, respectively. He received his Ph.D. degree in electrical and computer engineering from the Broadband and Wireless Networking Laboratory, School of Electrical and Computer Engineering, Georgia Institute of Technology, Atlanta, in 2004. He is currently a professor with the Department of Electrical and Electronics Engineering, Koc University and director of the NWCL. His current research interests are in wireless communications, bio-inspired communications, nano-scale and molecular communications, and information theory. He is an Associate Editor of IEEE Transactions on Vehicular Technology, International Journal of Communication Systems (Wiley), and Nano Communication Networks Journal (Elsevier). He is currently General Co-Chair of ACM MOBICOM 2012, IEEE MoNaCom 2012, and TPC CoChair of IEEE ISCC 2012. He is a member of ACM. He received the IBM Faculty Award twice, in 2010 and 2008, and the Turkish Academy of Sciences Distinguished Young Scientist Award 2008 (TUBA-GEBIP). 